A ANTROPOLOC

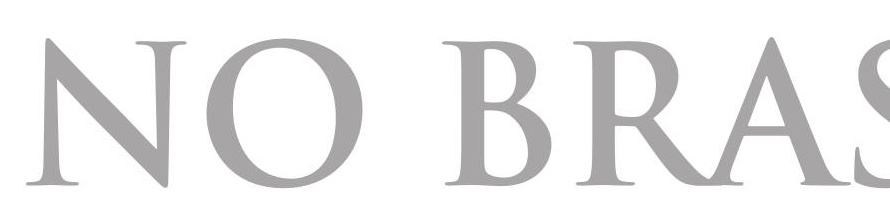

E A INTERDISCIPLINARID

POSSÍ 


\section{A ANTROPOLOGIA NO BRASIL: É A INTERDISCIPLINARIDADE POSSÍVEL?}

\section{F R A N C I S C O M. S A L Z A N O}




\title{
Resumo
}

A história da Antropologia no Brasil foi dividida em três fases: 1) Os pioneiros, 2) Período formativo, e 3) Fase contemporânea. Os principais eventos e exemplos de figuras paradigmáticas foram apresentados com relação aos dois primeiros períodos, enquanto para o terceiro foi fornecida uma lista de todos os presidentes da Associação Brasileira de Antropologia. A seguir desenvolveu-se abordagem similar para as quatro subáreas em que a Antropologia é tradicionalmente subdividida: Antropologia Social/Cultural, Arqueologia, Linguística, e Antropologia Física/Biológica. A pergunta feita no final é se a interação entre essas subáreas é possível. A resposta é de que uma abordagem verdadeiramente interdisciplinar é difícil, mas parece ser o melhor caminho para pesquisas de ponta. Sugere-se, portanto, uma abertura maior dos Programas de PósGraduação em Antropologia brasileiros à contratação de especialistas nas quatro subáreas, através de montagens de projetos de cunho nitidamente interdisciplinares.

Palavras-chave: História da Antropologia, Antropologia no Brasil, Interdisciplinaridade.

\begin{abstract}
The history of Anthropology in Brazil was divided in three phases: 1) The pioneers, 2) Formative period, and 3) Contemporaneous phase. The main events and examples of paradigmatic persons were presented for the two first periods, while for the third a list of all the presidents of the Brazilian Association of Anthropology was provided. A similar approach was followed in relation to the subareas in which Anthropology is traditionally subdivided: Social/Cultural Anthropology, Archaeology, Linguistics, and Physical/Biological Anthropology. The question asked at the end was whether the interaction among these subareas is possible. The answer is that a really interdisciplinary approach is difficult, but seems to be the best way for frontier research. Therefore, a suggestion is made that Brazilian Postgraduate Programs in Anthropology should seek specialists in the four subareas, through the establishment of clearly interdisciplinary projects.
\end{abstract}

Keywords: History of Anthropology, Anthropology in Brazil, Interdisciplinarity. 


\section{Resumen}

La historia de Antropología en Brasil fue dividida en tres etapas: 1) Los pioneros, 2) Período formativo, y 3) Fase contemporánea. Los principales eventos y ejemplos de figuras paradigmáticas fueran presentados con relación a los dos primeros períodos, mientras cuanto al tercer fue presentada una lista de todos los presidentes de la Asociación Brasileña de Antropología. Después un abordaje similar fue hecho para las cuatro subáreas en que la Antropología es tradicionalmente subdividida: Antropología Social/Cultural, Arqueología, Lingüística, y Antropología Física/ Biológica. La pregunta hecha al final fue si la interacción entre esas subáreas es posible. La respuesta es que un abordaje verdaderamente interdisciplinario es difícil, pero ese parece ser el mejor camino para investigaciones de punta. Se hace una sugerencia, por lo tanto, para una mayor apertura de los Programas de Posgrado en Antropología brasileños para la contratación de especialistas en las cuatro subáreas, por medio de proyectos netamente interdisciplinarios.

Palabras claves: Historia de la Antropología, Antropología en Brasil, Interdisciplinaridad. 


\section{MORIBUNDA OU PLENA DE VIDA?}

Etimologicamente Antropologia significa o estudo do homem (ou da espécie humana) e em seu sentido lato envolve a análise comparativa tanto de sua biologia quanto de manifestações culturais no que se refere ao tempo, variedade, lugar e condição (Comas 1966). Devido a essa amplitude não têm faltado críticos à disciplina ou seus cultores, e Gustavo Lins Ribeiro, presidente da Associação Brasileira de Antropologia ( $\mathrm{ABA}$ ) na gestão 2002-2004 declarou que "A Antropologia pode ser pensada como uma fênix, cuja morte ou agonia prolongada tem sido anunciada várias vezes, ao menos desde a década de 1920" (Ribeiro 2006:107). No entanto, esse mesmo autor salientou que dos dois mil antropólogos calculados por Kroeber (1953) existirem em sua época, chega-se atualmente a uma estimativa conservadora de pelo menos $30 \mathrm{mil}$.

Durham (2006) salientou que a Antropologia de hoje, como a de ontem, é relevante porque teve e tem um profundo sentido revolucionário em termos da visão de mundo que esteve e ainda está se construindo em nossa sociedade. Ela enfatizou a relação dialética do reconhecimento das diferenças como um requisito para a interpretação correta do conceito de humanidade comum. Outros aspectos abordados por ela e muitos outros autores é o do grau com que a atividade antropológica deve estar vinculada com as reivindicações sociais dos grupos que estuda. Essas implicações éticas, quando se consideram grupos tribais, foram avaliadas de maneira ampla em Salzano \& Hurtado (2004).

\section{CARACTERÍSTICAS}

No seu sentido estrito o termo Antropologia refere-se ao estudo da história natural da espécie humana, isto é, da Antropologia Física ou Biológica. Já no seu sentido lato, indicado na seção anterior, a palavra tem um âmbito mais abrangente, que inclui a Antropologia Cultural ou Social, a Arqueologia e a Linguística, além da Antropologia Biológica. Esse é o chamado enfoque dos quatro campos, particularmente adotado nos Estados Unidos da América (Spencer 1997:ix). Tradicionalmente a Antropologia no Brasil tem seguido um rumo diverso, os Departamentos de Antropologia concentrando primariamente cultores da Antropologia Cultural, com grupos geralmente menores de arqueólogos. A participação de linguistas e antropólogos biológicos nessas unidades é praticamente nula.

A proposta de um novo Programa de Pós-Graduação nessa área, a ser desenvolvido na Universidade Federal do Pará, é a de justamente modificar este panorama, com uma abordagem interdisciplinar que contemple essas quatro subdisciplinas. É dentro deste contexto que apresentarei, nas seções seguintes, um panorama muito geral sobre a História da Antropologia no Brasil. Deve ficar claro que, como a minha formação é basicamente na área biológica, haverá um inevitável viés na minha versão dessa história. Mas como sou associado da ABA há quase meio século, e as pesquisas que desen- 
volvo em populações indígenas e nãoindígenas da América do Sul envolvem sempre abordagens que incluem a cultura, a arqueologia e a linguística, creio que a abordagem não será muito inapropriada. Ela está focada em figuras e eventos paradigmáticos; portanto, peço de antemão desculpas pela eventual omissão de fatos ou figuras que seriam considerados essenciais em outros contextos.

\section{HISTÓRIA DA ANTROPOLOGIA NO BRASIL - OS PIONEIROS}

A Primeira Fase da História da Antropologia no Brasil pode ser convenientemente delimitada entre 1835, com a descoberta por Peter W. Lund (1801-1880) do material ósseo de Lagoa Santa e 1933 (imediatamente antes da fundação da Universidade de São Paulo, em 1934). Os nove nomes listados no Quadro 1 correspondem à figura clássica do antropólogo da época, que investigava todos os aspectos da Antropologia em seu sentido lato. Desses, apenas cinco eram brasileiros ou tinham residência permanente no Brasil: Barbosa Rodrigues e Curt Nimuendaju no norte, Nina Rodrigues na Bahia, e von Ihering e Roquete Pinto respectivamente em São Paulo e Rio de Janeiro. Ehrenreich, Ranke e Koch-Grünberg, de nacionalidade alemã, realizaram expedições de estudos, de caráter temporário, de nossos indígenas.

Quadro 1

História da Antropologia no Brasil. Fase 1: Os pioneiros (1835-1933)

\begin{tabular}{lcl}
\hline \multicolumn{1}{c}{ Personagens } & Datas & \multicolumn{1}{c}{ Natureza do estudo } \\
\hline P.W. Lund & $1835-1844$ & Estudos paleoantropológicos em Lagoa Santa \\
J. Barbosa Rodrigues & $1872-1899$ & Etnologia e arqueologia \\
P. Ehrenreich & $1887-1897$ & Expedições de estudo de indígenas brasileiros \\
R. Nina Rodrigues & $1890-1904$ & Estudos africanistas \\
K.E. Ranke & $1898-1907$ & Expedições de estudo de indígenas brasileiros \\
H. von Ihering & $1898-1914$ & Investigações de caráter geral \\
T. Koch-Grünberg & $1903-1904$ & Etnologia indígena \\
Curt Nimenendaju & $1914-1933$ & Etnologia e arqueologia \\
E. Roquete Pinto & $1917-1942$ & Investigações de caráter geral \\
\hline
\end{tabular}

Fonte: Castro Farias (1952); Salzano (1997).

HISTÓRIA DA ANTROPOLOGIA NO BRASIL - PERÍODO FORMATIVO

Todos os historiadores de ciência concordam em que a fundação da Universidade de São Paulo (USP) foi marco decisivo para a institucionalização da pesquisa científica no Brasil. Schwartzman (1979) descreveu em detalhe como surgiu e se concretizou a idéia, que envolvia como ponto fundamental a criação da Faculdade de Filosofia, Ciências e Letras, destinada a formar 
a elite nacional. O trabalho de estruturação da USP e as decisões cruciais relativas à mesma foram obra basicamente de três pessoas: Julio de Mesquita Filho, diretor do jornal Estado de São Paulo; Armando Salles de Oliveira, interventor federal; e Paulo Duarte, intelectual e político.

Desde seu início a nova instituição estabeleceu um critério de valorização da excelência que permanece até hoje. Foi realizado ativo recrutamento de intelectuais, especialmente na Europa, e o exemplo foi seguido, pelo menos parcialmente, em outras regiões do país.

O Quadro 2 lista, por ordem alfabética, algumas figuras paradigmáticas que desenvolveram estudos entre 1934 e 1954, antes da fundação da ABA. A contribuição estrangeira é exemplificada pela presença de dois antropólogos franceses (Alfred Métraux, Roger Bas- tide) e dois norte-americanos (Charles Wagley, Donald Pierson). Entre os brasileiros ou com residência permanente no país a maioria, naturalmente, é de São Paulo (Schaden, Willems, Fernandes, Mussolini, Schultz, Baldus), mas há também representantes do Rio de Janeiro (Ramos, D. Ribeiro), Rio Grande do Sul (Laytano), nordeste (Cascudo, R. Ribeiro, Azevedo) e norte (Galvão, Arnaux) do Brasil. As referências bibliográficas indicadas no Quadro nem sempre indicam a obra mais importante das personagens listadas, servindo apenas como exemplos de trabalhos aos quais tive acesso. Entretanto, o livro de Arnaud (1989) documenta bem sua trajetória de 50 anos de estudos em populações indígenas; e o de Corrêa (1987) fornece uma sinopse do período 1930-1960, com testemunhos e bibliografias de Donald Pierson e Emílio Willems.

Quadro 2

História da Antropologia no Brasil. Fase 2: Período Formativo (1934-1954)

\begin{tabular}{ll}
\hline \multicolumn{1}{c}{ Algumas figuras paradigmáticas listadas em ordem alfabética } \\
\hline 1. Alfred Métraux & 10. Expedito Arnaux \\
2. Arthur Ramos & 11. Florestan Fernandes \\
3. Charles Wagley & 12. Gioconda Mussolini \\
4. Dante de Laytano & 13. Harald Schultz \\
5. Darcy Ribeiro & 14. Herbert Baldus \\
6. Donald Pierson & 15. Luís da Câmara Cascudo \\
7. Eduardo Galvão & 16. René Ribeiro \\
8. Egon Schaden & 17. Roger Bastide \\
9. Emilio Willems & 18. Thales de Azevedo \\
\hline
\end{tabular}

Fonte: Ramos (1943/47); Métraux (1946); Fernandes (1949); Laytano (1956); Ribeiro (1957); Galvão (1960); Schultz (1963); Schaden (1965); Mussolini (1969); Waglevy (1971); Azevedo (1975); Corrêa (1987); Arnaud (1989). 
HISTÓRIA DA ANTROPOLOGIA NO BRASIL - FASE CONTEMPORÂNEA

Seria impossível, no espaço disponível, cobrir de maneira adequada o que ocorreu na Antropologia do país neste último meio século. Decidi, portanto, listar (Quadro 3) todos os presidentes da ABA, que sem dúvida fornecem uma amostra adequada de personagens importantes na área. Os dois períodos indicados na tabela relacionam-se à transformação da ABA de uma instituição fundamentalmente elitista, com fortes exigências de ingresso, para uma associação mais democrática em que os estudantes de graduação e pós-graduação também teriam espaço. A distribuição das instituições às quais os presidentes estão ou estavam vinculados reflete o que ocorre na ciência brasileira em geral; cerca da metade das instituições (11/24 gestões, $46 \%$ ) localiza-se no sudeste, cinco no Distrito Federal, cinco no sul e três no nordeste. Quase a totalidade dos presidentes da ABA podem ser classificados como antropólogos sociais/culturais; as exceções são três, que pelo menos parcialmente podem ser categorizados como antropólogos físicos/biológicos (Castro Faria, Loureiro Fernandes, Thales de Azevedo) e uma que é lingüista (Yonne Leite).

Quadro 3

História da Antropologia no Brasil. Fase 3: Contemporânea (1955-Presente)

Os presidentes da Associação Brasileira de Antropologia ${ }^{1}$

I. Na estrutura clássica

$\begin{array}{ll}1955 & \text { Luiz de Castro Faria (MN) } \\ 1958 & \text { José Loureiro Fernandes (UFPR) } \\ 1959 & \text { Darcy Ribeiro (UFRJ) } \\ 1961 & \text { Herbert Baldus (MP) } \\ 1963 & \text { Eduardo Galvão (UnB) } \\ 1966 & \text { Manuel Diégues Jr. (UFPR) } \\ 1974 & \text { Thales de Azevedo (UFBA) } \\ 1976 & \text { René Ribeiro (UFPE) }\end{array}$

II. Pós-revolução dos jovens
1978 Luis de Castro Faria (MN)
1980 Eunice Durham (USP)
1982 Gilberto C. Alves Velho (MN)
1984 Roberto Cardoso de Oliveira (UnB)
1986 Manuela Carneiro da Cunha (USP)
1988 Antonio A. Arantes (UNICAMP)
1990 Roque de Barros Laraia (UnB)
1992 Silvio Coelho dos Santos (UFSC)
1994 João Pacheco de Oliveira (MN) 


$\begin{array}{ll}1996 & \text { Mariza Corrêa (UNICAMP) } \\ 1998 & \text { Yonne Leite (MN) } \\ 2000 & \text { Ruben Oliven (UFRGS) } \\ 2002 & \text { Gustavo Lins Ribeiro (UnB) } \\ 2004 & \text { Miriam Pillar Grossi (UFSC) } \\ 2006 & \text { Luís R. Cardoso de Oliveira (UnB) } \\ 2008 & \text { Carlos Caroso (UFBA) }\end{array}$

${ }^{1}$ Chave para as siglas: MN: Museu Nacional; MP: Museu Paulista; UFBA: Universidade Federal da Bahia; UFPE: Universidade Federal de Pernambuco; UFPR: Universidade Federal do Paraná; UFRGS: Universidade Federal do Rio Grande do Sul; UFRJ: Universidade Federal do Rio de Janeiro; UFSC: Universidade Federal de Santa Catarina; UNICAMP: Universidade Estadual de Campinas; UnB: Universidade de Brasília; USP: Universidade de São Paulo.

Fonte: Corrêa (2003); Eckert \& Godoi (2006); Trajano Filho \& Ribeiro (2004) apresentaram um panorama muito abrangente desta fase, com ênfase nos Programas de Pós-Graduação em Antropologia, iniciados em 1971.

\section{AS SUBÁREAS - ANTROPOLOGIA SOCIAL/CULTURAL}

O Quadro 4 fornece nove exemplos selecionados de obras importantes desenvolvidas na subárea da Antropologia Social/Cultural. Três delas (as de Ramos, Baldus e Ribeiro) são de caráter geral, considerando tanto o panorama brasileiro quanto uma tentativa de interpretação de todo o processo civilizatório; o estudo do contato interétnico está muito bem representado com quatro contribuições (Pierson, Cardoso, Cardoso de Oliveira, Santos); enquanto que Galvão e Maybury-Lewis concentraram-se em populações indígenas.

Quadro 4

Exemplos selecionados de obras importantes em Antropologia Social/Cultural no Brasil ${ }^{1}$

\begin{tabular}{lll}
\hline \multicolumn{1}{c}{ Ano } & \multicolumn{1}{c}{ Autor } & \multicolumn{1}{c}{ Título } \\
\hline 1942 & Donald Pierson & Negroes in Brazil: A Study of Race Contact at Bahia \\
$1943 / 1947$ & Arthur Ramos & Introdução à Antropologia Brasileira \\
1954,1968 & Herbert Baldus & Bibliografia Crítica da Etnologia Brasileira \\
1960 & Eduardo Galvão & Áreas Culturais Indígenas do Brasil - 1900-1959 \\
1962 & Fernando H. Cardoso & Capitalismo e Escravidão no Brasil Meridional \\
1964 & Roberto C. de Oliveira & O Índio e o Mundo dos Brancos \\
1967 & David Maybury-Lewis & Akwê-Shavante Society \\
1968 & Darcy Ribeiro & O Processo Civilizatório \\
1973 & Silvio C. dos Santos & Índios e Brancos no Sul do Brasil \\
\hline
\end{tabular}

${ }^{1}$ Informações completas sobre as obras são fornecidas na bibliografia. Ver também Amorim (2001) e Sampaio Silva (2007), que descrevem as vidas e as obras de respectivamente Roberto Cardoso de Oliveira e Eduardo Galvão 


\section{AS SUBÁREAS - ARQUEOLOGIA}

Exemplos selecionados de eventos e personagens importantes para a Arqueologia no Brasil estão listados no Quadro 5. Os estudos têm um passado respeitável, que pode retroagir a 1870. Mas o primeiro programa nacional ocorreu apenas na década de 1960, através de uma interação muito profícua entre Mário Simões, do Museu Paraense Emílio Goeldi, e Clifford Evans e Betty Meggers, da Smithsonian Institution (Washington, DC, EUA), com o apoio do Conselho Nacional de Desenvolvimento Científico e Tecnológico (CNPq). Os estudos abrangeram todo o país e forneceram uma base preciosa de informações que posteriormente foram ampliadas com pesquisas especialmente do casal Emperaire, Pedro I. Schmitz, André Prous e Oldemar Blasi no centro-oeste, sudeste e sul do Brasil. Mais recentemente Anna Roosevelt vem desenvolvendo pesquisa muito abrangente na Amazônia.

\section{AS SUBÁREAS - LINGUÍSTICA}

As cerca de 180 línguas indígenas faladas no Brasil, metade das quais conta com população de até 500 indivíduos (quatro etnias têm população superior a 20 mil) são a principal ponte entre linguistas e antropólogos. Listas de palavras das mesmas foram coletadas desde praticamente a época do descobrimento do país pelos europeus, mas o estudo científico detalhado dessas línguas é ainda escasso. Como indicado no Quadro 6, a principal figura na área é Arion Rodrigues, que há cerca de meio século vem estudando, sistematizando, e incentivando a pesquisa das línguas dos indígenas brasileiros.

Quadro 5

Eventos e personagens importantes para a Arqueologia no Brasil

\begin{tabular}{|c|c|c|}
\hline Ano & Autor & Título \\
\hline 1870 & Arqueologia na Ilha de Marajó & Ferreira Penna e J.B. Steere \\
\hline $1879-1892$ & Antiguidades do Amazonas & João Barbosa Rodrigues \\
\hline $1950-1952$ & $\begin{array}{l}\text { Teses na Universidade da Columbia } \\
\text { sobre a arqueologia do Território do } \\
\text { Amapá e Ilha do Marajó }\end{array}$ & Clifford Evans e Betty J. Meggers \\
\hline $1962-1969$ & Escavações em sambaquis & Paulo Duarte (1899-1984) \\
\hline $1965-1970$ & $\begin{array}{l}\text { Programa Nacional de Pesquisas } \\
\text { Arqueológicas (PRONAPA) }\end{array}$ & C. Evans, B.J. Meggers, Mario Simões \\
\hline $1950-1970$ & Estudos em Lagoa Santa e outros sítios & $\begin{array}{l}\text { Joseph Emperaire (falec.: 1955) } \\
\text { e Annete Laming-Emperaire (1917-1977) }\end{array}$ \\
\hline 1972 & Programa Arqueológico de Goiás & Pedro I. Schmitz \\
\hline $1960-1990$ & $\begin{array}{l}\text { Pesquisas diversas sobre a Arqueologia } \\
\text { no sudeste e sul }\end{array}$ & $\begin{array}{l}\text { André Prous, José P. Brochado, Oldemar } \\
\text { Blasi, Ondemar Ferreira Dias Jr. }\end{array}$ \\
\hline $1990-2000$ & Arqueologia amazônica & Anna C. Roosevelt \\
\hline
\end{tabular}

Fonte: Simões (1967,1974); Duarte (1968, 1970); Mendes (1970); Penteado (1978); Schmitz et al. (1986); Carvalho (1987); Prous (1992); Roosevelt et al. (2002). 


\section{Quadro 6}

Aspectos relacionados com a Lingüística e sua relação com a Antropologia no Brasil

1. A princial conexão entre a Lingüística e a Antropologia envolve, no Brasil, o estudo das línguas indígenas.

2. Elementos do Summer Institute of Linguistics, em sua ação doutrinadora, têm trabalhado com grande número de línguas indígenas, e informações a respeito podem ser obtidas no livro Ethnologue, organizado por Barbara F. Grimes.

3. A principal figura desta área no Brasil é, sem dúvida, Aryon D’All'Igna Rodrigues, que há cerca de meio século vem estudando, sistematizando, e incentivando o estudo das línguas de nossos indígenas.

4. Outras personagens importantes, listadas por ordem alfabética são: Ana Suelly A.C. Cabral, Denny Moore, Ernesto Magliazza, Greg Urban, Lucy Seki e Yonne Leite.

Fonte: Rodrigues (1986); Grimes (1992): Cabral \& Rodrigues (2007).

Quadro 7

A Antropologia Biológica no Brasil. Fase 1: Os pioneiros (1835-1933)

\begin{tabular}{lcl}
\hline \multicolumn{1}{c}{ Personagens } & Datas & \multicolumn{1}{c}{ Natureza do estudo } \\
\hline $\begin{array}{l}\text { J.B. Lacerda, } \\
\text { J.R. Peixoto }\end{array}$ & $1875-1893$ & $\begin{array}{l}\text { Morfologia de indígenas brasileiros } \\
\text { (vivos e extintos) }\end{array}$ \\
$\begin{array}{l}\text { A. Bovero, R. Locchi, } \\
\text { A. Fróes da Fonseca }\end{array}$ & $1914-1940$ & Anatomia étnica, São Paulo e Rio de Janeiro \\
$\begin{array}{l}\text { Olympio Fonseca Filho } \\
\text { J. Bastos de Ávila, }\end{array}$ & $1924-1970$ & Parasitologia étnica \\
M.J. Pourchet & $1931-1983$ & Antropometria comparada, crescimento físico \\
\hline
\end{tabular}

Fonte: Castro Farias (1952); Salzano (1997). 


\section{Quadro 8}

A Antropologia Biológica no Brasil. Fase 2: Período Formativo (1934-1954)

\begin{tabular}{lcl}
\hline \multicolumn{1}{c}{ Personagens } & Datas & \multicolumn{1}{c}{ Natureza do estudo } \\
\hline $\begin{array}{l}\text { O. Machado de Souza } \\
\text { Gioconda Mussolini }\end{array}$ & $1934-1943$ & Estudos anatômicos, classificação tipológica \\
Ernani M. da Silva & $1944-1969$ & $\begin{array}{l}\text { Etnomedicina, aculturação } \\
\text { Grupos sanguíneos e siclemia em indígenas }\end{array}$ \\
$\begin{array}{ll}\text { Noel Nutels, } \\
\text { J.A.N. Miranda }\end{array}$ & $1947-1997$ & $\begin{array}{l}\text { Epiemiologia e efeitos da turbeculose } \\
\text { em indígenas brasileiros }\end{array}$ \\
$\begin{array}{l}\text { Newton Freire-Maia, } \\
\text { O. Frota-Pessoa }\end{array}$ & $1952-1989$ & \begin{tabular}{l} 
Estrutura populacional em neo-brasileiros \\
\hline
\end{tabular}
\end{tabular}

Fonte: Castro Farias (1952); Salzano \& Freire-Maia (1970); Salzano (1997).

Quadro 9

A Antropologia Biológica no Brasil. Fase 3: Contemporânea (1955-Presente)

\begin{tabular}{|c|c|c|}
\hline Personagens & Datas & Natureza do estudo \\
\hline F.M. Salzano e equipe & 1958-presente & $\begin{array}{l}\text { Estrutura populacional, marcadores genéticos, } \\
\text { mistura interétnica }\end{array}$ \\
\hline M.C. Mello e Alvim & $1962-1997$ & Antropologia física pré-histórica \\
\hline $\begin{array}{l}\text { R.G. Baruzzi, } \\
\text { J.P.B Vieira Filho }\end{array}$ & 1970-presente & Investigações médicas em indígenas brasileiros \\
\hline $\begin{array}{l}\text { Luiz F. Ferreira, } \\
\text { Adauto G. Araujo }\end{array}$ & 1980-presente & Parasitologia de grupos pré-históricos \\
\hline $\begin{array}{l}\text { H. Schneider, } \\
\text { M.P.C. Schneider, } \\
\text { S.E.B. Santos, } \\
\text { J.F. Guerreiro, } \\
\text { A.K.C. Ribeiro-dos-Santos }\end{array}$ & 1981-presente & $\begin{array}{l}\text { Estrutura populacional, marcadores genéticos, } \\
\text { mistura interétnica na Amazônia }\end{array}$ \\
\hline Walter A. Neves e equipe & 1981-presente & $\begin{array}{l}\text { Antropologia física pré-histórica, biologia óssea, } \\
\text { adaptação }\end{array}$ \\
\hline $\begin{array}{l}\text { M.A. Zago, } \\
\text { M.L. Petzl-Erler, } \\
\text { S.D.J. Pena }\end{array}$ & 1983-presente & $\begin{array}{l}\text { Marcadores genéticos em indígenas } \\
\text { e neo-brasileiros }\end{array}$ \\
\hline
\end{tabular}

Fonte: Castro Farias (1952); Salzano \& Freire-Maia (1970); Salzano (1997). 


\section{AS SUBÁREAS - ANTROPOLOGIA FÍSICA/BIOLÓGICA}

Os Quadros 7 a 9 listam, nas três fases já estabelecidas para a Antropologia em geral, exemplos selecionados de estudos nessa subárea. É importante salientar que todas essas pesquisas (com exceção das desenvolvidas no Museu Nacional e dos trabalhos de Gioconda Mussolini) foram realizadas fora dos Departamentos de Antropologia, como decorrência da estrutura institucional atualmente vigente no país. Iniciadas por estudos morfológicos, elas incluíram posteriormente a variação protéica, e mais recentemente o estudo do DNA, o material genético, em indivíduos sadios, indígenas, e neobrasileiros. Porém, também existem estudos importantes de paleoantropologia e de antropologia médica, tanto de populações atuais quanto pré-históricas.

\section{É A INTERDISCIPLINARIDADE POSSÍVEL?}

É a interdisciplinaridade possível? Cardoso de Oliveira (2006), comparando os Programas de Pós-Graduação em Antropologia e em Ciências Sociais, sugeriu que a interdisciplinaridade parecia ter perdido o encantamento dos primeiros tempos. E ele apresentou duas hipóteses para explicar isso: (a) O "atavismo" disciplinar, a dificuldade em superar as barreiras da formação especializada; e (b) O caráter intervencionista das políticas públicas brasileiras, que atualmente favorecem estudos com metas muito definidas. $\mathrm{O}$ mesmo autor diferenciou multidisciplinaridade de interdisciplinaridade. No primeiro caso o que há é a convivência paralela em um espaço físico ou intelectual de áreas diversas; no segundo a atuação em um espaço marcado pela tensão epistêmica, onde as diferentes disciplinas convivem em diálogo permanente. Segundo Cardoso de Oliveira, é neste segundo caminho que se desenvolvem mais facilmente pesquisas de ponta.

Se existe estudo no qual a interdisciplinaridade é considerada essencial, este é o da evolução biológica, e mais especificamente o da evolução humana. Devido ao seu caráter essencialmente sintetizador de fatos diversos, essas investigações se contrapõem à tendência reducionista que predomina na biologia hoje. Especialmente na nossa espécie as influências de fatores sócioculturais, médico-epidemiológicos e de estrutura demográfica necessariamente devem ser consideradas em qualquer interpretação do nosso processo genético-evolucionário.

\section{CONCLUSÃO}

A Antropologia, em seus primórdios, foi basicamente interdisciplinar e integradora. Com o acúmulo de novas informações, de caráter diverso, tem havido uma tendência cada vez maior para a especialização em determinada subárea. No caso do Brasil, os Programas de Pós-Graduação em Antropologia estão muito mais voltados à Antropologia Social ou Cultural do que às outras subdisciplinas. Não está na hora de revertermos esta tendência?

\section{NOTAS}

${ }^{1}$ As pesquisas do autor são financiadas pelo Conselho Nacional de Desenvolvimento 
Científico e Tecnológico, Programas Institutos do Milênio e Apoio a Grupos de Excelência e Fundação de Amparo à Pesquisa do Estado do Rio Grande do Sul.

\section{REFERÊNCIAS}

Amorim, M.S. 2001. Roberto Cardoso de Oliveira um artífice da antropologia. Brasília: Paralelo 15 e Coordenação de Aperfeiçoamento de Pessoal de Nível Superior.

Araújo, A.J.G. \& L.F. Ferreira (Orgs.). 1992. Paleopatologia, paleoepidemiologia. Estudos multidisciplinares. Rio de Janeiro: Escola Nacional de Saúde Pública.

Arnaud, E. 1989. O indio e a expansão nacional. Belém: CEJUP.

Azevedo, T. 1975. Democracia racial. Ideologia e realidade. Petrópolis: Vozes.

Baldus, H. 1954. Bibliografia crítica da etnologia brasileira. Volume I. São Paulo: XXXI. Congresso Internacional de Americanistas.

Baldus, H. 1968. Bibliografia crítica da etnologia brasileira. Volume II. Hannover: Münstermann - Druck.

Baruzzi, R.G., L.F. Marcopito, M.L.C. Serra, F.A.A. Souza \& C. Stabile. 1977. The Kren-Akorore: a recently contacted indigenous tribe, in Health and disease in tribal societies. Editado por P. Hugh-Jones, pp. 179-211. Amsterdam: Elsevier.

Cabral, A.S.A.C. \& A.D. Rodrigues (Orgs.). 2007. Linguas e culturas Tupi. Campinas: Editora Curt Nimuendajú e Laboratório de Línguas Indígenas, Universidade de Brasília.

Cardoso, F.H. 1977. Capitalismo e escravidão no Brasil meridional. $\mathrm{O}$ negro na sociedade escravocrata do Rio Grande do Sul. Rio de Janeiro: Paz e Terra.

Cardoso de Oliveira, R. 1964. O indio e o mundo dos brancos. A situação dos Tukúna do Alto Solimões. São Paulo: Difusão Européia do Livro.
Cardoso de Oliveira, R. 2006. Antropologia e interdisciplinaridade, in Homenagens. Associação Brasileira de Antropologia 50 anos. Organizado por C. Eckert \& E.P. Godoi, pp. 51-56. Florianópolis: Nova Letra.

Carvalho, E. (Org.). 1987. A pesquisa do passado. Arqueologia no Brasil. Rio de Janeiro: Universidade do Estado do Rio de Janeiro.

Castro Faria, L. 1952. Pesquisas de Antropologia Física no Brasil. Boletim do Museu Nacional, Antropologia 13:1-106.

Comas, J. 1996. Manual de Antropología Fisica. México, D.F: Universidad Autónoma de México.

Corrêa, M. 1987. História da Antropologia no Brasil (1930-1960). Campinas: Vértice, Editora dos Tribunais.

Corrêa, M. 2003. As reuniōes brasileiras de Antropologia: cinquenta anos (1953-2003). Brasília: Associação Brasileira de Antropologia.

Duarte, P. (Org.). 1968. Pré-história brasileira. São Paulo: Instituto de Pré-História da Universidade de São Paulo. 1970. Estudos de pré-história geral e brasileira. São Paulo: Instituto de Pré-História da Universidade de São Paulo.

Durham, E.R. 2006. A relevância da Antropologia, in Homenagens. Associação Brasileira de Antropologia 50 anos. Organizado por C. Eckert \& E.P. Godoi, pp. 85-94. Florianópolis: Nova Letra.

Eckert, C. \& E.P. Godoi. (Orgs.). 2006. Homenagens. Associação Brasileira de Antropologia 50 anos. Florianópolis: Nova Letra.

Fernandes, F. 1949. A organização social dos Tupinambá. São Paulo: Instituto Progresso Editorial.

Galvão, E. 1960. Áreas culturais indígenas do Brasil-1900-1959. Boletim do Museu Paraense Emilio Goeldi, Antropologia 8:1-41.

Grimas, B.F. (Ed.). 1992. Ethnologue. Languages of the world. Dallas: Summer Institute of Linguistics. 
Kroeber, A.L. 1953. Introduction, in Anthropology today. Editado por A.L. Kroeber, pp. xiiixv. Chicago: University of Chicago Press. Laytano, D. 1956. Populações indígenas. Estudo histórico de suas condições atuais no Rio Grande do Sul - Caingang. Revista do Musen Júlio de Castilhos e Arquivo Histórico do Rio Grande do Sul 5(6):201-246.

Maybury-Lewis, D. 1967. Akw-Shavante society. Oxford: Clarendon Press.

Mendes, J.C. 1970. Conheça a pré-história brasileira. São Paulo: Polígono e Editora da Universidade de São Paulo.

Métraux, A. 1946. The Caingang, in Handbook of South American Indians, volume 1. Editado por J.H. Steward, pp. 445-475. Washington, D.C.: Smithsonian Institution.

Mussolini, G. Organizadora. 1969. Evolução, raça e cultura. São Paulo: Companhia Editora Nacional.

Neves, W.A., A.G.M. Araujo, R. Kipnis \& L.B. Piló. 2007. O projeto Origens e microevolução do homem na América: uma abordagem paleoantropológica, in Arqueologia e Patrimônio de Minas Gerais. Organizado por A.P.P.L. Oliveira, pp.73-90. Juiz de Fora: Universidade Federal de Juiz de Fora.

Penteado, A.R. 1978. Coletânea de estudos em homenagem a Annette Laming-Emperaire. São Paulo: Museu Paulista, Universidade de São Paulo.

Pierson, D. 1942. Negroes in Brazil: a study of race contact at Babia. Chicago: Chicago University Press.

Prous, A. 1992. Arqueologia brasileira. Brasília: Editora da Universidade de Brasília.

Ramos, A. 1943/1947. Introducão à Antropologia brasileira. Rio de Janeiro: Editora Casa do Estudante do Brasil.

Ribeiro, D. 1957. Culturas e linguas indígenas do Brasil. Educação e Ciências Sociais 2:5-102. 1968. Estudos de Antropologia da civilização. I. O processo civilizatório. Etapas da evolução sociocultural. Rio de Janeiro:
Civilização Brasileira.

Ribeiro, G.L. 2006. Pós-imperialismo, antropologias mundiais e a tensão provincianismo metropolitano/cosmopolitismo provinciano, in Homenagens. Associação Brasileira de Antropologia 50 anos. Organizado por C. Eckert \& E.P. Godoi, pp. 107-113. Florianópolis: Nova Letra.

Rodrigues, A.D. 1986. Linguas brasileiras. Para o conhecimento das linguas indígenas. São Paulo: Edições Loyola.

Roosevelt, A., J. Douglas \& L. Brown. 2002. The migrations and adaptations of the first Americans. Clovis and pre-Clovis viewed from South America, in The first Americans. The Pleistocene colonization of the New World. Editado por N.G. Jablonski, pp. 159-235. San Francisco: Ca-lifornia Academy of Sciences.

Salzano, F.M. 1997. Brazil, in History of physical anthropology. An encyclopedia, volume 1. Editado por F. Spencer, pp. 207-213. New York: Garland.

Salzano, F.M. \& M.C. Bortolini. 2002. The evolution and genetics of Latin American populations. Cambridge: Cambridge University Press.

Salzano, F.M. \& N. Freire-Maia. 1970. Problems in Human Biology. A study of Brazilian populations. Detroit: Wayne State University Press. Salzano, F.M. \& A.M. Hurtado. 2004. Lost paradises and the ethics of research and publication. New York: Oxford University Press.

Sampaio Silva, O. 2007. Eduardo Galvão: indios e caboclos. São Paulo: Annablume.

Santos, S.C. 1973. Índios e brancos no sul do Brasil - a dramática experiência dos Xokleng. Florianópolis: EDEME, Indústria Editorial e Gráfica.

Schaden, E. 1965. Aculturação indígena. Revista de Antropologia 13:1-315.

Schmitz, P.I., M.B. Ribeiro, A.S. Barbosa, M.O. Barbosa \& A.F. Miranda. 1986. Caiapônia. Arqueologia nos cerrados do Brasil Cen- 
tral. São Leopoldo: Unisinos, Universidade do Vale dos Sinos.

Schultz, H. 1963. Hombu. La vie des indiens dans la jungle brésilienne. Amsterdam/Rio de Janeiro: Colibris Editora.

Schwartzman, S. 1979. Formação da comunidade científica no Brasil. São Paulo: Companhia Editora Nacional, Financiadora de Estudos e Projetos (FINEP).

Simões, M.F. 1967. Programa Nacional de Pesquisas Arqueológicas. Resultados preliminares do primeiro ano (1965-1966). Belém: Museu Paraense Emílio Goeldi.

Simões, M.F. 1974. Programa Nacional de Pesquisas Arqueológicas. Resultados preliminares do quinto ano (1969-1970). Belém: Museu Paraense Emílio Goeldi.

Spencer, F. 1997. Preface, in History of physical anthropology. An encyclopedia, volume 1. Editado por F. Spencer, pp. ix. New York: Garland.

Trajano Filho, W. \& G.L. Ribeiro (Orgs). 2004. O campo da antropologia no Brasil. Rio de Janeiro: Contra Capa, Associação Brasileira de Antropologia.

Wagley, C. 1971. The formation of the American population, in The ongoing evolution of Latin American populations. Editado por F.M. Salzano, pp. 19-39. Springfield: Charles C Thomas. 